\title{
Analysis of Lateral Distribution of Atmospheric Cherenkov Light at High Mountain Altitude Towards Event Reconstruction
}

\author{
Alexander Mishev ${ }^{1,2}$ \\ ${ }^{1}$ Institute for Nuclear Research and Nuclear Energy, Bulgarian Academy of Sciences, 1784 Sofia, Bulgaria \\ ${ }^{2}$ Oulu Unit, Sodankyla Geophysical Observatory, University of Oulu, 90014 Oulu, Finland
}

Correspondence should be addressed to Alexander Mishev, alex_mishev@yahoo.com

Received 12 November 2012; Accepted 3 December 2012

Academic Editors: S. Alexeyev, L. Marek-Crnjac, O. A. Sampayo, and A. S. Tonachini

Copyright (C) 2012 Alexander Mishev. This is an open access article distributed under the Creative Commons Attribution License, which permits unrestricted use, distribution, and reproduction in any medium, provided the original work is properly cited.

\begin{abstract}
Air shower simulations with CORSIKA6.990 code using FLUKA 2011 and QGSJET II hadron generators are performed. The simulations are carried out at high mountain observation level, namely, Chacaltaya cosmic ray station. The lateral distribution of atmospheric Cherenkov light produced by various primary particles, namely, proton, helium, oxygen, and iron nuclei, is obtained over wide energy range, between $10^{11} \mathrm{eV}$ and $10^{17} \mathrm{eV}$. The lateral distribution is obtained, integrating over time and angle Cherenkov photons till $800 \mathrm{~m}$ from the shower axis. The shapes of the obtained distributions are compared and parameterized. Three different approximations of lateral distribution of Cherenkov light are compared. On the basis of inverse problem solution an event analysis, towards mass composition and energy reconstruction of the primary particle, is carried out. The applications and scientific potential for HECRE experiment proposal are discussed.
\end{abstract}

\section{Introduction}

Cosmic rays are high, ultra-high, and extremely high energy particles of extraterrestrial origin, which constantly impinge the Earth's atmosphere. The primary cosmic (CR)s rays extend over twelve decades of energy with the corresponding decline in the intensity. The flux goes down from $10^{4} \mathrm{~m}^{-2} \mathrm{~s}^{-1}$ at energies $\sim 10^{9} \mathrm{eV}$ to $10^{-2} \mathrm{~km}^{-2} \mathrm{yr}^{-1}$ at energies $\sim 10^{20} \mathrm{eV}$. The shape of the spectrum is featureless, with small deviation from the power law function across this large energy range. The observed change in the slope $\alpha E^{-2.7}$ to $\alpha E^{-3.0}$ around 1$3.10^{15} \mathrm{eV}$ is known as the "knee" of the spectrum. The major unsolved problems are related to origin and acceleration mechanisms of primary cosmic ray [1,2]. The study of primary cosmic ray mass composition in the energy range around the "knee" is of a crucial importance for 
understanding the origin of cosmic rays and their acceleration and propagation mechanism. As example, the change from light to heavier composition with increasing the energy of cosmic ray nuclei is related to energy limit of their acceleration in galactic sources.

It is regarded that the bulk of cosmic rays originate from the galaxy. The part below the "knee" comes from galactic supernovas, particles accelerated by the shocks in the supernova remnants (SNR). The explanation of the "knee" of the spectrum is based on the SNR diffuse shock acceleration mechanism limits $[3,4]$. In this model the galactic supernovas are the only galactic candidate with sufficient energy [5]. The supernova diffuse shock acceleration mechanism can produce high energy particles up to some maximal energy, which is limited by the lifetime of the shock wave. Other possibility is when the particles are so energetic and they can no be confined in the acceleration region [6]. The measurements of the individual cosmic ray spectrum and the precise estimation of mass composition are very important in order to obtain detailed information about the sources of primary cosmic ray and build an adequate model of cosmic ray origin and acceleration mechanisms [7].

While the low-energy CR particles are just absorbed in the atmosphere, those with energies greater then $1 \mathrm{GeV} /$ nucleon generate new particles through interactions with atomic nuclei in air-an atmospheric cascade. An extensive air shower (EAS) usually consists of billions of secondary particles (mostly electrons and muons) that arrive at ground level over large areas. The predominant interactions are electromagnetic; the cross-section for the production of hadron and muon pairs are several orders of magnitude smaller than that for electron-pair production. In the electromagnetic shower, protons produce electron-positron pairs, and electrons and positrons produce photons via Bremsstrahlung. The relativistic charged particles in the shower generate Cherenkov light.

Above $10^{14} \mathrm{eV}$ the only possibility for cosmic ray detection and measurement is ground based, that is, the detection of one or several of the components of secondary cosmic ray. One of the most convenient techniques is the atmospheric Cherenkov technique, that is, the detection of the Cherenkov light in extensive air showers. The atmospheric Cherenkov technique is based on registration of the very brief flash of Cherenkov radiation generated by the cascade of relativistic charged particles produced when a very high-energy CR particle impinge the atmosphere. The Cherenkov light generated in EAS was observed by Galbraith and Jelley in 1953 [8]. The Whipple telescope detected Crab Nebula $[9,10]$ on the basis of imaging the air shower.

Using measurements with atmospheric Cherenkov telescopes the mass composition of primary CR could be estimated [11,12]. The detection of the air Cherenkov light at ground level by an array of telescopes or photomultipliers is convenient tool for all particle energy spectrum [13] measurement, as was recently reported [14,15]. Such types of telescopes are based on time and angle integration of Cherenkov light flux at given observation level.

Recently a new calibration experiment for a primary CR mass composition estimation in the "knee" region was proposed [16, 17]. The HECRE [18] experiment proposal, situated at high mountain altitude of $516 \mathrm{~g} \mathrm{~cm}^{-2}$ observation level is with general aim mass composition and energy spectrum studies of primary CR around the "knee" region. A significant part of this experiment is the Cherenkov array. Moreover, the existing air shower array at Chacaltaya was recently completed with Cherenkov detectors [19]. The detailed simulation of Cherenkov array of HECRE experiment is the principal aim of this study in attempt to study the experiment capability for event reconstruction. 


\section{Lateral Distribution of Atmospheric Cherenkov Light}

In this study the evolution of atmospheric cascade, respectively, the lateral distribution of atmospheric Cherenkov light, is carried out with the CORSIKA 6.990 code [20] with corresponding hadron interaction models FLUKA 2011 [21] and Quark Gluon String with JETs QGSJET II [22]. COsmic Ray SImulations for KASKADE (CORSIKA) code is the most widely used atmospheric cascade simulation tool. The code simulates the interactions and decays of various nuclei, hadrons, muons, electrons, and photons in the atmosphere. The particles are tracked through the atmosphere until they undergo reactions with an air nucleus or in the case of unstable secondary particles, they decay. The result of the simulations is detailed information about the type, energy, momenta, location, and arrival time of the produced secondary particles at given selected altitude a.s.l. The primary particles that can be considered are protons, light, middle and heavy nuclei up to Iron.

The observation level was assumed to $516 \mathrm{~g} \mathrm{~cm}^{-2}$, which corresponds to Chacaltaya CR station. The US standard atmosphere was assumed during the simulations. This observation level is near to shower maximum, especially for the hadronic cascades with energies around the "knee". As a result the fluctuations of shower development are not as important compared with lower observation levels. This permits to obtain flatter distributions of the different shower components, namely, atmospheric Cherenkov light flux during the simulations. The simulated particles are primary proton, iron, helium, and oxygen nuclei. One large detector $800 \times 800 \mathrm{~m}$ was considered during the simulations, in attempt to collect the quasi-totality of Cherenkov photons in the shower. As a result a significant reduce of the statistical fluctuations is achieved. As a results the obtained lateral distribution of Cherenkov light flux densities in EAS is with less uncertainties, comparing to previous works [23, 24], where a grid of detectors was used.

Figures 1 and 2 present the Cherenkov light in EAS produced by primary protons over the energy ranges $10^{11}-10^{13} \mathrm{eV}$ and $10^{13}-10^{17} \mathrm{eV}$. The obtained lateral distributions of atmospheric Cherenkov light due to various cosmic ray nuclei below and in the region of the "knee" are presented in Figures 3 and 4. The lateral distributions of Cherenkov light flux densities in EAS initiated by primary nuclei, namely, helium and oxygen, are between the generated by proton and iron nuclei. An analysis of fluctuations of Cherenkov light flux as a function of the bin size is carried out. In a low energy range till $5.10^{12} \mathrm{eV}$ the statistical fluctuations are strongly dependent of the bin size, which is considered for analysis. In this energy range it is necessary to increase the obtained statistical fluctuations in the case of small detectors, for example, photomultipliers with Winston cones. In this study this is related only for primary proton nuclei. In a high-energy range above $10^{13} \mathrm{eV}$ the fluctuations due to the longitudinal development of the shower dominate. Therefore the fluctuations above this energy are not related to the bin size.

\section{Parameterization of the Lateral Distribution and Event Reconstruction}

The obtained lateral distributions are approximated with various parameterizations [25-27]. Generally the reconstruction methods are based on a given assumption and parameterization of lateral distribution of Cherenkov light in EAS. In this connection the convenient parameterization is crucial for the event analysis and reconstruction of primary particle 


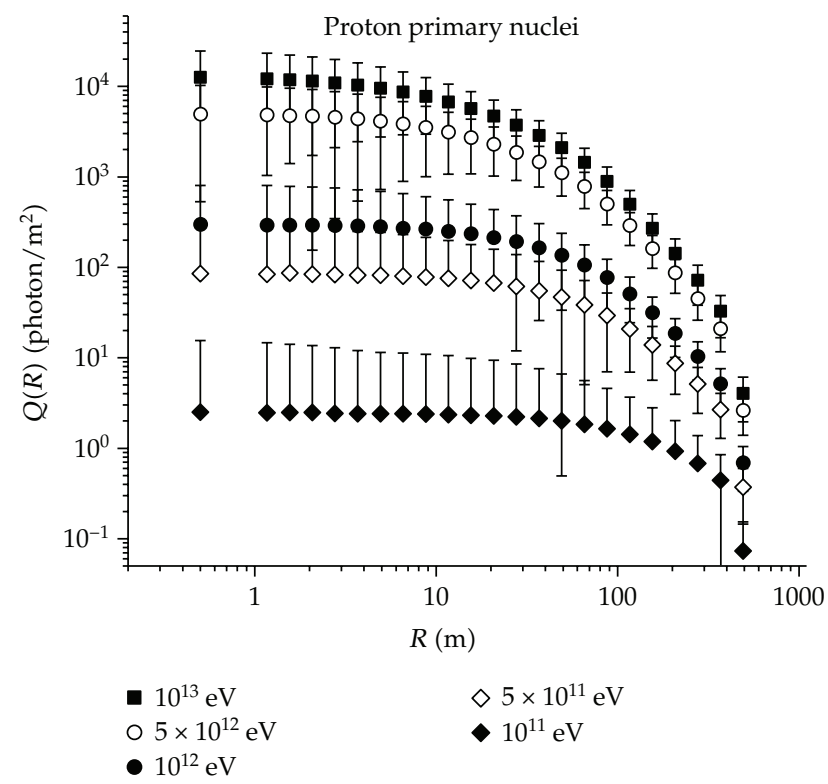

Figure 1: Lateral distribution of Cherenkov light flux in EAS produced by primary proton showers in the energy range 1011-1013 eV at Chacaltaya observation level.

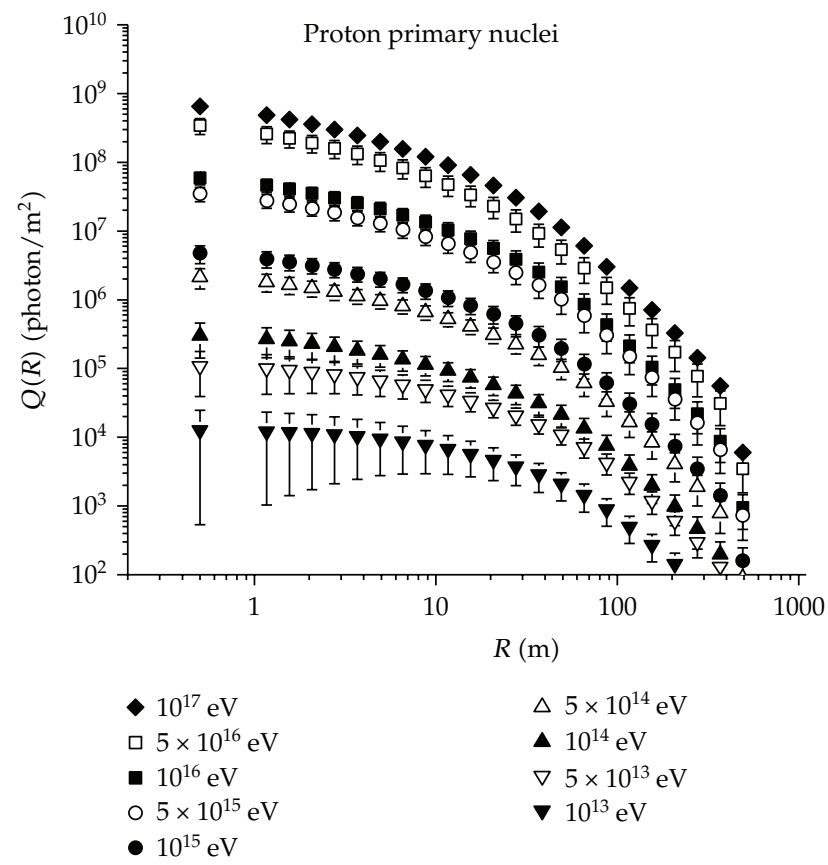

Figure 2: Lateral distribution of Cherenkov light flux in EAS produced by primary proton showers in the energy range 1013-1017 eV at Chacaltaya observation level. 


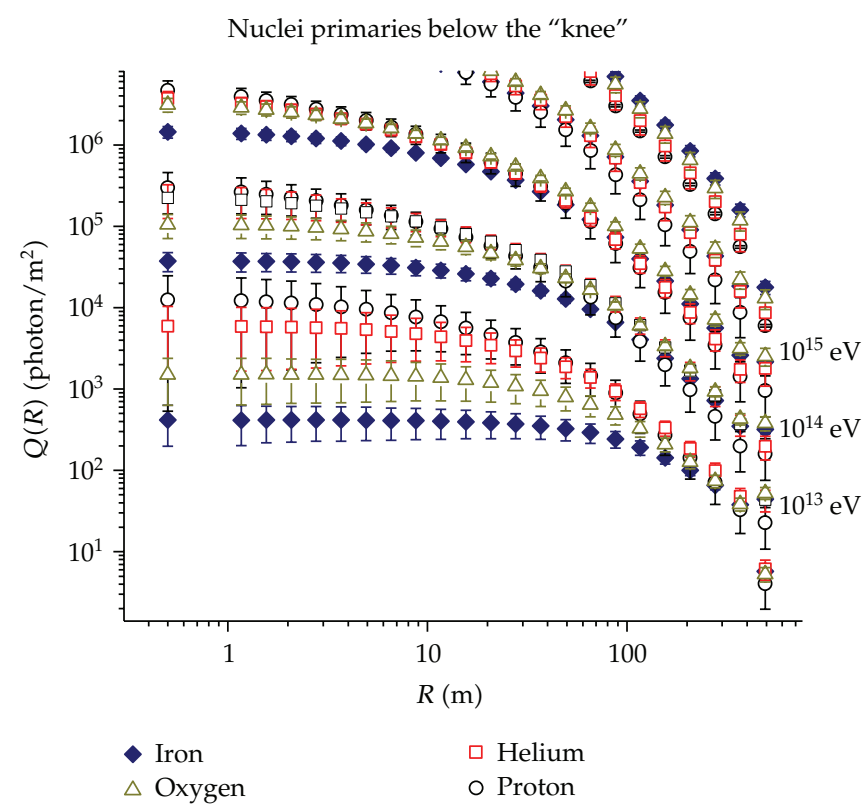

Figure 3: Lateral distribution of Cherenkov light flux in EAS produced by various nuclei below the "knee" at Chacaltaya observation level.

characteristics $[16,17,28,29]$. The results for various primary protons approximations are plotted in Figure 5. The proposed parameterization for HECRE experiment,

$$
Q(R)=\frac{\sigma e^{a} e^{-\left[R / \gamma+\left(R-r_{0}\right) / \gamma+(R / \gamma)^{2}+\left(\left(R-r_{0}\right) / \gamma\right)^{2}\right]}}{\gamma\left[(R / \gamma)^{2}+\left(\left(R-r_{0}\right) / \gamma\right)^{2}+R \sigma^{2} / \gamma\right]}
$$

is also applied for approximation of lateral distribution of atmospheric Cherenkov light produced by proton, helium, oxygen and iron nuclei. In (3.1) where $R$ is the distance from the shower axis, and $\sigma, \gamma, a$, and $r_{0}$, are model parameters. The results are presented in Figures $6,7,8$, and 9 .

The approximation (3.1) was successfully applied for various problems, namely, gamma hadron separation at lower observation depths [30, 31], fast Monte Carlo simulation of the detector response of wave front sampling and angle integration Cherenkov arrays [32], mass composition and cosmic ray energy spectrum estimation for Tunka array [33, 34].

The obtained lateral distributions, with corresponding parameterizations, are used for study of the event analysis capability of HECRE experiment proposal. A large amount of simulated events with CORSIKA code was used. In general the method for event reconstruction is based on inverse problem solution [35] assuming given parameterization and subsequent integration of the reconstructed lateral distribution. The solution of the inverse problem is carried out with afxy-code $[36,37]$ using Levenberg-Marquardt numerical algorithm [38, 39]. In Figure 10 is presented the obtained accuracy for energy estimation as a function of the distance from the center of detector array using various approximations. The parameterizations $[26,27]$ demonstrate similar results at distance till $150 \mathrm{~m}$ from center of the detector array. The primary particle energy is reconstructed with $10-15 \%$ accuracy. The 


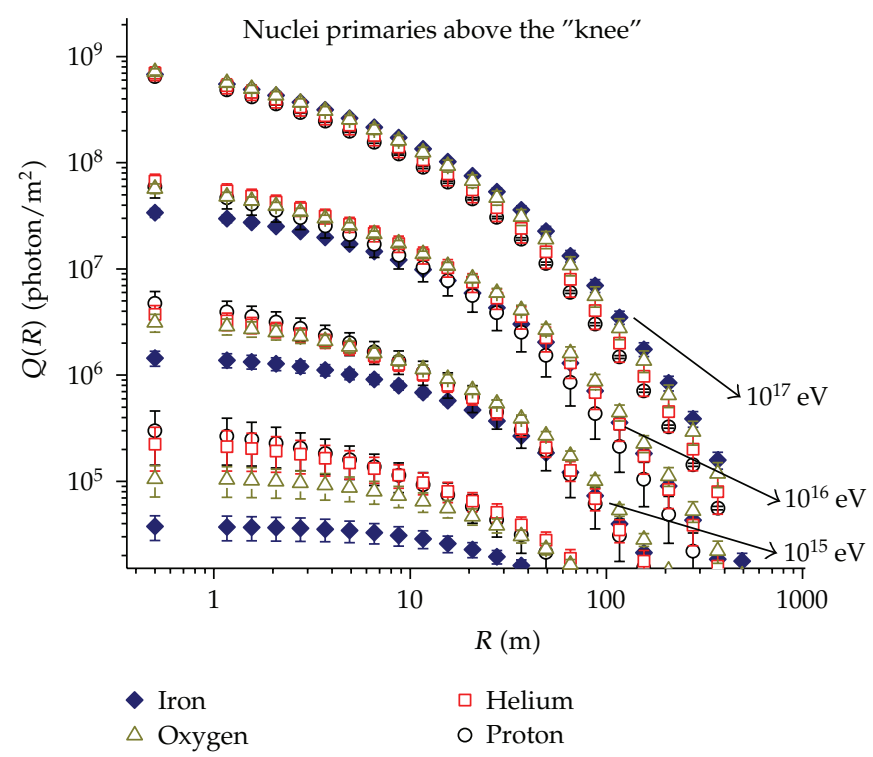

Figure 4: Lateral distribution of Cherenkov light flux in EAS produced by various nuclei around the "knee" at Chacaltaya observation level.

Table 1: Reconstruction efficiency for various primary particles for various distance from detector array.

\begin{tabular}{lcccc}
\hline & $50 \mathrm{~m}$ & $100 \mathrm{~m}$ & $150 \mathrm{~m}$ & $>150 \mathrm{~m}$ \\
\hline All particles & 0.812 & 0.774 & 0.73 & 0.7 \\
Protons & 0.81 & 0.773 & 0.72 & 0.69 \\
He, O and Fe & 0.83 & 0.85 & 0.81 & 0.73 \\
Rejection & 0.95 & 0.94 & 0.91 & 0.89 \\
\hline
\end{tabular}

application of parameterization [25] leads to primary particle energy reconstruction accuracy of $20 \%$ for distance of $50 \mathrm{~m}$ from detector array center and $50 \%$ for distance of $100 \mathrm{~m}$. At greater distance the parameterization [25] is not applicable, since the inverse problem is not solved. The parameterizations $[25,26]$ demonstrate greater accuracy within detector array. In addition they could be applied also for peripheric event reconstruction. At greater distance, outside of the detector array, the parameterization [26] leads to better accuracy in energy reconstruction.

An additional analysis for mass composition reconstruction is carried out. On the basis of a realistic cosmic ray spectrum [1] simulated with CORSIKA code and inverse problem solution, the atomic number (mass) of the reconstructed events is estimated. This analysis is possible only with parameterization [26]. Different model parameters corresponding to different primaries.

The result of this analysis is shown in Figure 11. Increasing the energy of the primary particle the accuracy for mass reconstruction also increases (lower model parameter uncertainty). The reconstruction efficiency as a function of the distance from detector array is summarized in Table 1.

The events within detector array are reconstructed with efficiency of about $80 \%$, with greater probability for middle and heavy nuclei because the flatter distributions. The rejection between different particles is nearly $100 \%$ (for reconstructed events). Only events with large 


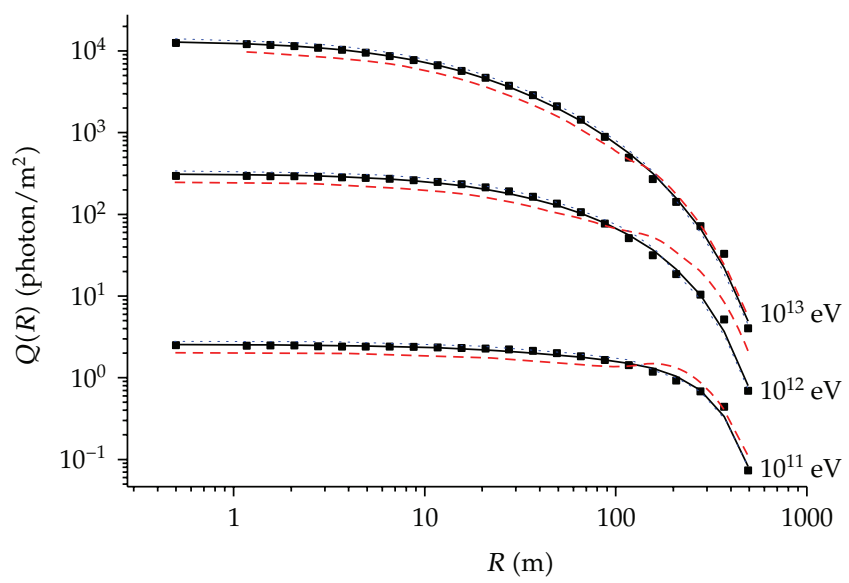

- Simulated data

- Mavrodiev et al., approximation, Nucl. Instr. Meth.

$$
\text { A } 530 \text { (2004) p.359 }
$$

..... Budnev et al., approximation, Proc. of 29th ICRC, Pune, India v6 p. 257

- - Anokhina et al., approximation, Astroph. and Space Science 209 (1993) p. 19

Figure 5: Various parameterization of atmospheric Cherenkov light in EAS.

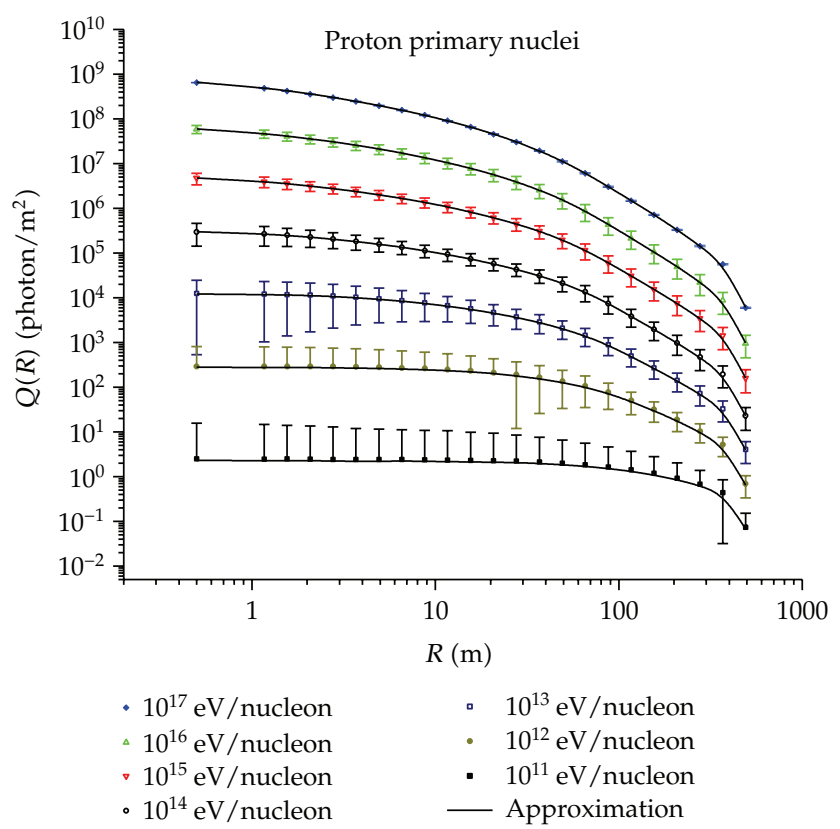

Figure 6: The approximation of obtained lateral distribution of atmospheric Cherenkov light in EAS produced by primary protons (scatter simulated data, line parameterization [26]). 


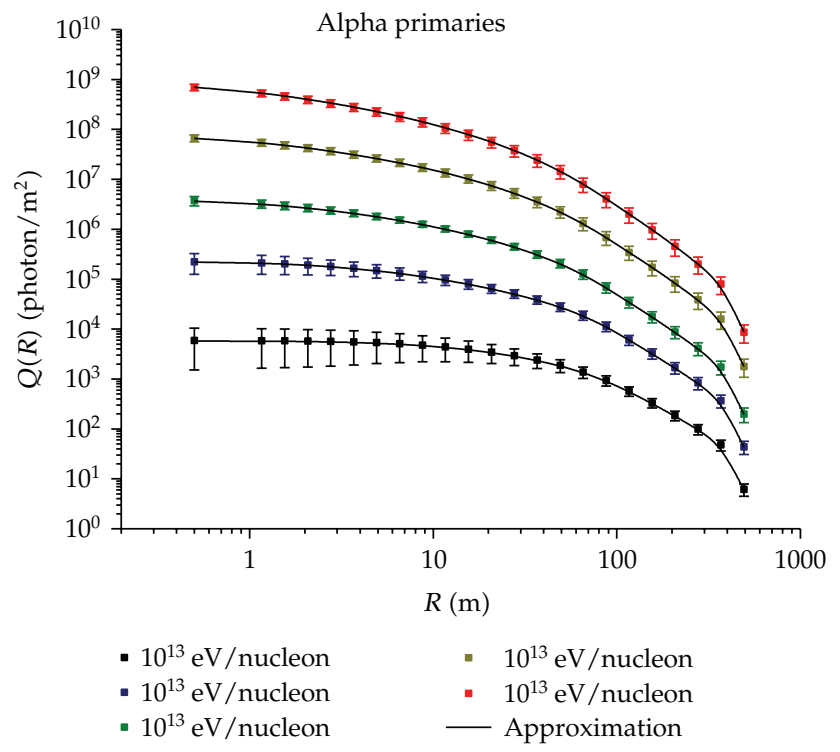

Figure 7: The approximation of obtained lateral distribution of atmospheric Cherenkov light in EAS produced by primary alpha particles (scatter simulated data, line parameterization [26]).

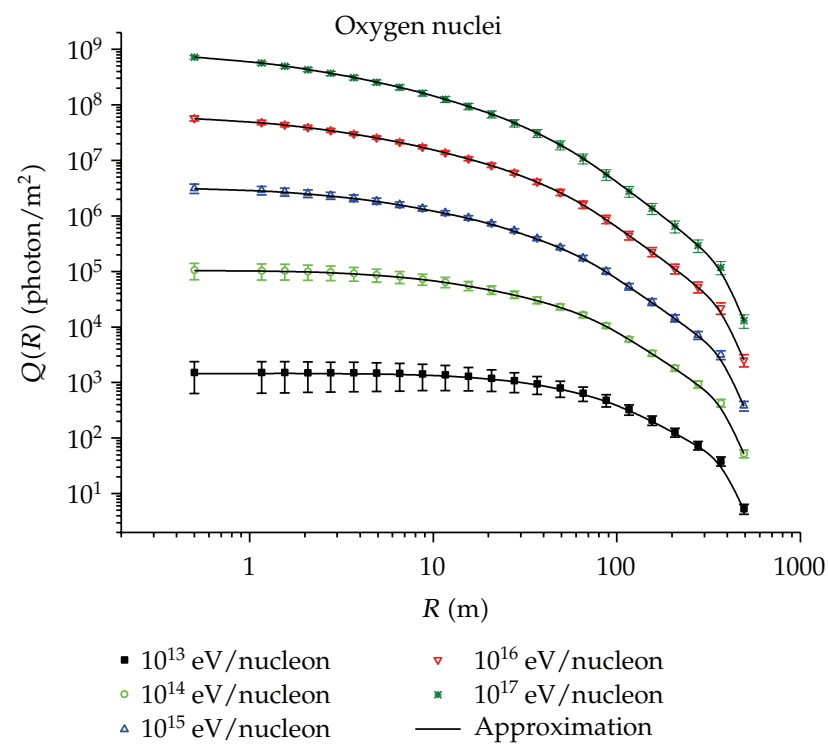

Figure 8: The approximation of obtained lateral distribution of atmospheric Cherenkov light in EAS produced by primary Oxygen nuclei (scatter simulated data, line parameterization [26]).

intrinsic fluctuations are not reconstructed, as well as very inclined events or some events outside of the detector array. As was expected the reconstruction efficiency for peripheric or events, which are outside of the detector array diminish. 


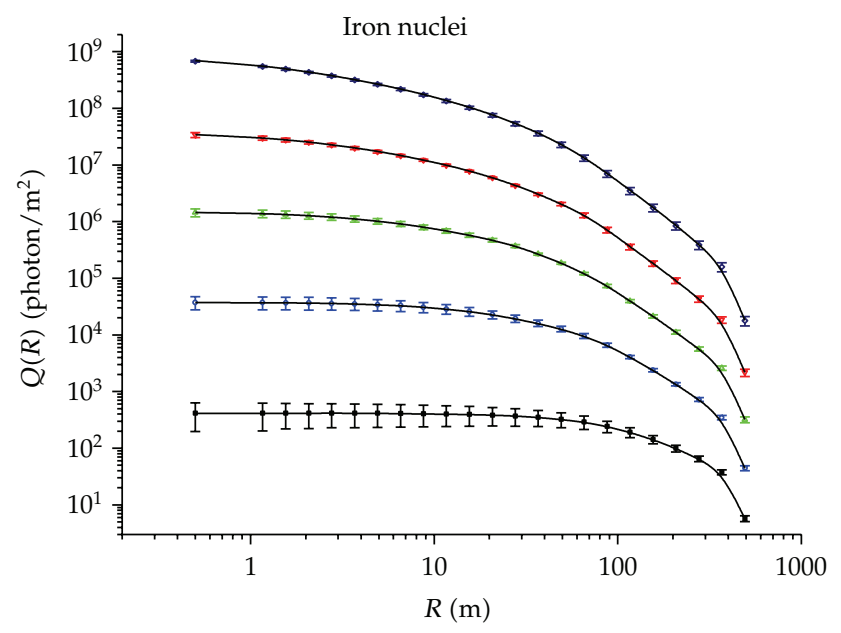

$\begin{array}{ll}-10^{13} \mathrm{eV} / \text { nucleon } & \text { - } 10^{13} \mathrm{eV} / \text { nucleon } \\ -10^{13} \mathrm{eV} / \text { nucleon } & \quad 10^{13} \mathrm{eV} / \text { nucleon } \\ -10^{13} \mathrm{eV} / \text { nucleon } & - \text { Approximation }\end{array}$

Figure 9: The approximation of obtained lateral distribution of atmospheric Cherenkov light in EAS produced by primary Iron nuclei (scatter simulated data, line parameterization [26]).

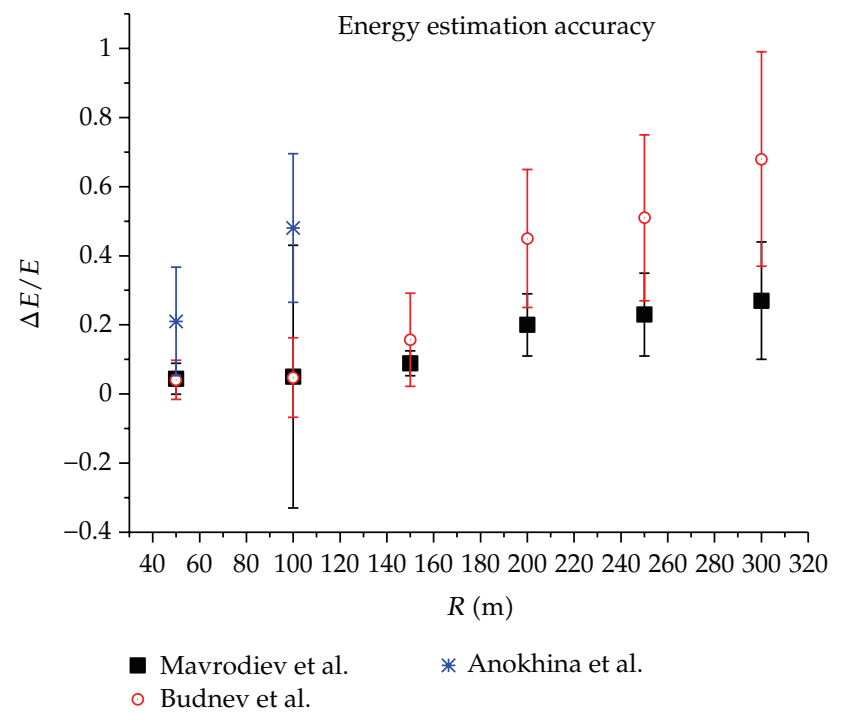

Figure 10: Accuracy for primary particle energy reconstruction using various parameterizations.

\section{Summary and Discussion}

Monte Carlo simulations have become an essential tool for the design and optimization of new or in development atmospheric Cherenkov telescopes. In this connection air shower simulations using CORSIKA6.990 code with FLUKA 2011 and QGSJETII hadron generators are performed. The lateral distribution of atmospheric Cherenkov light is obtained in a wide energy range for various primary particles, namely, proton, helium, oxygen, and iron 


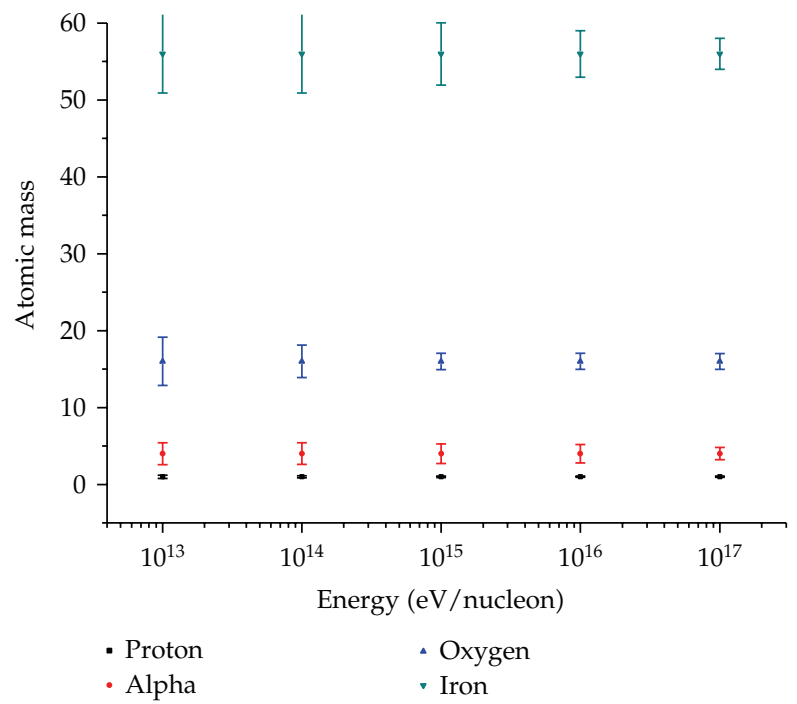

Figure 11: Primary mass reconstruction as a function of the energy of the primary particle.

nuclei. Three different parameterizations of atmospheric Cherenkov light lateral distribution at Chacaltaya observation level are compared. Their application for event reconstruction for HECRE experiment proposal is studied. It is demonstrated that parameterizations [26, 27] are both applicable for event analysis, namely, estimation of primary particle spectrum, since the previously proposed parameterization [25] could be used with several limitations. It is demonstrated that the initial goals of HECRE experiment proposal can be achieved with available methodology and technology. In addition the parameterization [26] gives the possibility for mass composition estimation of primary CR in the region of the "knee".

The proposed method and parameterization could be applied for different observation levels for wave front sampling Cherenkov arrays [40-42] as well as for multicomponent analysis [43] of EAS.

In addition the obtained lateral distributions demonstrate the capability of recently proposed method for mass composition estimation of primary cosmic ray based on inverse problem solution, which is in the frame of a new calibration experiment at high mountain observation level.

\section{Acknowledgments}

The author warmly acknowledges V. Guenchev, S. Piperov, E. Puncheva, and P. Konstantinov from High-energy Division of Institute for Nuclear Research and Nuclear Energy, Bulgarian Academy of Sciences, for giving computational time and technical assistance during the computations.

\section{References}

[1] K. Nakamura and (Particle Data Group), "Review of particle physics," Journal of Physics G, vol. 37, Article ID 075021, 2010.

[2] T. Gaisser, Cosmic Rays and Particle Physics, Cambridge University Press, 1990. 
[3] G. Krymsky, "A regular mechanism for the acceleration of charged particles on the front of a shock wave," Doklady Akademii Nauk USSR, vol. 234, pp. 1306-1308, 1977.

[4] R. Blandford and J. Ostriker, "Particle acceleration by astrophysical shocks," Astrophysical Journal, vol. 221, pp. L29-L32, 1978.

[5] R. Schlickeiser, "Cosmic-ray transport and acceleration," Astrophysical Journal, vol. 90, no. 2, pp. 929936, 1994.

[6] V.S. Ptuskin and V. N. Zirakashvili, "Limits on diffusive shock acceleration in supernova remnants in the presence of cosmic-ray streaming instability and wave dissipation," Astronomy and Astrophysics, vol. 403, no. 1, pp. 1-10, 2003.

[7] V. Berezinsky, "Transition from galactic to extragalactic cosmic rays," in Proceedings of the 30th International Cosmic Ray Conference, vol. 6, pp. 21-33, Merida, Mexico, 2009.

[8] W. Galbraith and J. V. Jelley, "Light pulses from the night sky associated with cosmic rays," Nature, vol. 171, no. 4347, pp. 349-350, 1953.

[9] G. Vacanti, M. F. Cawley, E. Colombo et al., "Gamma-ray observations of the Crab Nebula at TeV energies," Astrophysical Journal Letters, vol. 377, no. 2, pp. 467-479, 1991.

[10] T. Weekes, "Very high energy gamma-ray astronomy," Physics Reports, vol. 160, no. 1-2, pp. 1-121, 1988.

[11] J. Cortina, F. Arqueros, and E. Lorenz, "Methods for determining the primary energy of cosmic-ray showers," Journal of Physics G, vol. 23, no. 11, pp. 1733-1749, 1997.

[12] A. V. Plyasheshnikov, A. K. Konopelko, F. A. Aharonian, M. Hemberger, W. Hofmann, and H. J. Völk, "Study of mass composition and energy spectrum of primary cosmic radiation by the imaging atmospheric Cherenkov technique," Journal of Physics G, vol. 24, no. 3, pp. 653-672, 1998.

[13] A. Lindner, "A new method to reconstruct the energy and determine the composition of cosmic rays from the measurement of Cherenkov light and particle densities in extensive air showers," Astroparticle Physics, vol. 8, no. 4, pp. 235-252, 1998.

[14] B. K. Lubsandorzhiev, "TUNKA-EAS Cherenkov experiment in the Tunka Valley," Nuclear Instruments and Methods in Physics Research A, vol. 595, no. 1, pp. 73-76, 2008.

[15] V. V. Prosin, N. M. Budnev, O. A. Chvalaiev et al., "The cosmic ray mass composition in the energy range $10^{15}-10^{18} \mathrm{eV}$ measured with the tunka array: results and perspectives," Nuclear Physics B, vol. 190, no. C, pp. 247-252, 2009.

[16] J. Procureur and J. N. Stamenov, "Primary mass composition investigations at energies $10^{4}-10^{7} \mathrm{GeV}$ and selection of EAS at mountain altitudes," Nuclear Physics B, vol. 39, no. 1, pp. 242-250, 1995.

[17] J. Procureur and J. N. Stamenov, "Proposal for a new calibration experiment at Chacaltaya for a primary mass estimation in the "knee" region," Nuclear Physics B, vol. 52, no. 3, pp. 285-287, 1997.

[18] O. Saavedra and L. Jones, "Chacaltaya: towards a solution of the knee....?" Il Nuovo Cimento C, vol. 24, pp. 497-506, 2001.

[19] Y. Tsunesada, F. Kakimoto, H. Matsumoto et al., "A Cherenkov light detection at Mount Chacaltayato study nuclear composition of cosmic rays," in Proceedings of the 31th International Cosmic Ray Conference, Lodz, Poland, 2009.

[20] D. Heck, J. Knapp, J. N. Capdevielle, G. Schatz, and T. Thouw, “CORSIKA: a Monte Carlo code to simulate extensive air showers," Forschungszentrum Karlsruhe Report FZKA 6019, 1998.

[21] G. Battistoni, S. Muraro, P. R. Sala et al., "The FLUKA code: description and benchmarking," in Proceedings of the Hadronic Shower Simulation Workshop, M. Albrow and R. Raja, Eds., vol. 896 of AIP Conference Proceeding, pp. 31-49, September 2007.

[22] S. Ostapchenko, "QGSJET-II: towards reliable description of very high energy hadronic interactions," Nuclear Physics B, vol. 151, no. 1, pp. 143-146, 2006.

[23] F. Arqueros, A. Karle, E. Lorenz, S. Martinez, R. Plaga, and M. Rozanska, "Separation of gamma and hadron initiated air showers with energies between 20 and 500 TeV," Astroparticle Physics, vol. 4, no. 4, pp. 309-332, 1996.

[24] C. E. Portocarrero and F. Arqueros, "On the Cherenkov light produced at several observatory altitudes by extensive air showers in the energy range 0.01-10 TeV," Journal of Physics G, vol. 24, no. 1, pp. 235$253,1998$.

[25] A. M. Anokhina, V. I. Galkin, K. V. Mandritskaya, and T. M. Roganova, "Air shower Cerenkov radiation as an instrument for very high energy $\gamma$-astronomy (I)," Astrophysics and Space Science, vol. 209, no. 1, pp. 19-38, 1993.

[26] S. C. Mavrodiev, A. L. Mishev, and J. N. Stamenov, "A method for energy estimation and mass composition determination of primary cosmic rays at the Chacaltaya observation level based on 
the atmospheric Cherenkov light technique," Nuclear Instruments and Methods in Physics Research A, vol. 530, no. 3, pp. 359-366, 2004.

[27] N. Budnev, D. Chernov, O. Gress et al., "Cosmic ray energy spectrum and mass composition from $10^{15}$ to $10^{17} \mathrm{eV}$ by data of the tunka EAS cherenkov array," in Proceedings of the 29th International Cosmic Ray Conference, vol. 6, pp. 257-260, Pune, India, 2005.

[28] D. Himmelblau, Applied Nonlinear Programming, Mcgraw-Hill, 1972.

[29] W. H. Press, S. A. Teukolsky, W. T. Vetterling, and B. P. Flannery, Numerical Recipes: The Art of Scientific Computing, Cambridge University Press, 3rd edition, 2007.

[30] A. Mishev, S. Mavrodiev, and J. Stamenov, "Primary cosmic ray studies based on atmospheric cherenkov light technique at high-mountain altitude," in Frontiers in Cosmic Ray Research, pp. 25-82, Nova Science, 2007.

[31] A. Mishev, S. Mavrodiev, and J. Stamenov, "Gamma rays studies based on atmospheric Cherenkov technique at high mountain altitude," International Journal of Modern Physics A, vol. 20, no. 29, pp. 7016-7019, 2005.

[32] A. Mishev and J. Stamenov, "Cherenkov telescope for ground based gamma astronomy kartalska field project proposal," Journal of American Institute of Physics, vol. 899, p. 548, 2007.

[33] A. A. Al-Rubaiee, O. A. Gress, K. S. Lokhtin, Y. V. Parfenov, and S. I. Sinegovskii, "Modeling and parameterization of the spatial distribution of Čerenkov light from extensive air showers," Russian Physics Journal, vol. 48, no. 10, pp. 1004-1011, 2005.

[34] A. A. Al-Rubaiee and S. I. Sinegorskii, "Determination of the type and energy of a cosmic ray particle based on the spatial distribution function of Čeerenkov light from extensive air showers," Russian Physics Journal, vol. 49, no. 6, pp. 673-675, 2006.

[35] L. Alexandrov, S. C. Mavrodiev, A. Mishev, and J. Stamenov, "Estimation of the primary cosmic radiation characteristics," in Proceedings of the 27th International Cosmic Ray Conference, pp. 257-261, Hamburg, Germany, August 2001.

[36] L. Aleksandrov, "Regularized Newton-Kantorovič computing processes," Journal of Computational Mathematics and Mathematical Physics, vol. 11, pp. 36-43, 1971.

[37] L. Alexandrov and A. Mishev, "Application of afxy-code for parameterization of ionization yield function $\mathrm{Y}$ in the atmospherefor primary cosmic ray protons," http://arxiv.org/abs/0712.3174.

[38] K. Levenberg, "A method for the solution of certain non-linear problems in least squares," Quarterly of Applied Mathematics, vol. 2, pp. 164-168, 1944.

[39] D. W. Marquardt, "An algorithm for least-squares estimation of nonlinear parameters," Journal of the Society For Industrial and Applied Mathematics, vol. 11, pp. 431-441, 1963.

[40] B. A. Antokhonov, D. Besson, S. F. Beregnev et al., "A new $1 \mathrm{~km}^{2}$ EAS Cherenkov array in the Tunka Valley," Nuclear Instruments and Methods in Physics Research A, vol. 639, no. 1, pp. 42-45, 2011.

[41] A. A. Ivanov, S. P. Knurenko, Z. E. Petrov, M. I. Pravdin, and I. Y. Sleptsov, "Enhancement of the Yakutsk array by atmospheric Cherenkov telescopes to study cosmic rays above $10^{15} \mathrm{eV}$," Astrophysics and Space Sciences Transactions, vol. 6, no. 1, pp. 53-57, 2010.

[42] M. Tluczykont, D. Hampf, D. Horns et al., "The ground-based large-area wide-angle gamma-ray and cosmic-ray experiment HiSCORE," Adavances in Space Research, vol. 48, pp. 1935-1941, 2011.

[43] S. P. Swordy and D. B. Kieda, "Elemental composition of cosmic rays near the knee by multiparameter measurements of air showers," Astroparticle Physics, vol. 13, no. 2-3, pp. 137-150, 2000. 

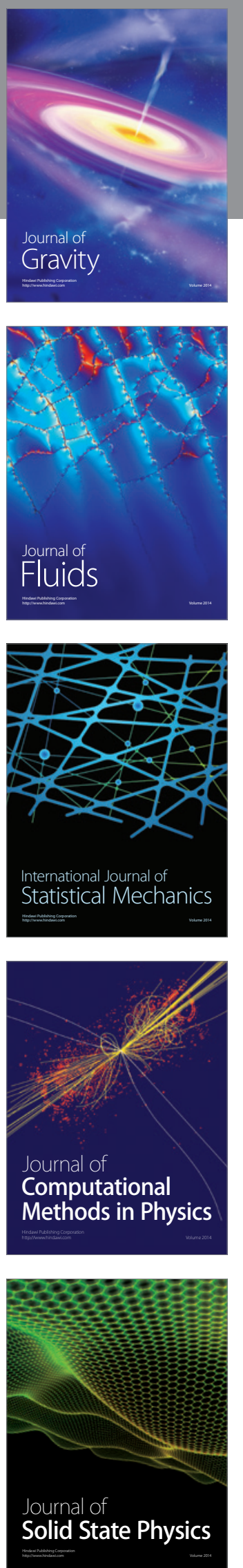
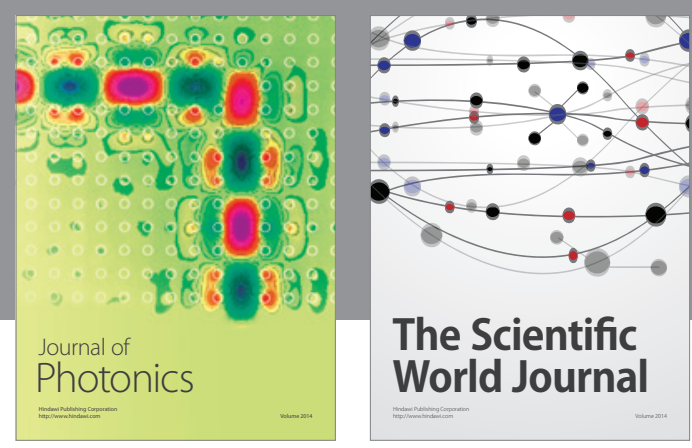

The Scientific World Journal

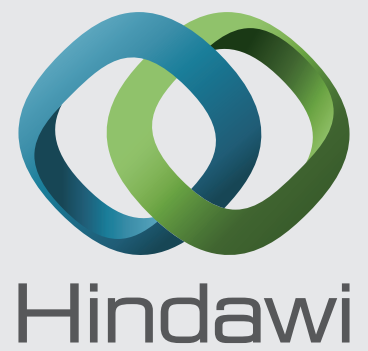

Submit your manuscripts at http://www.hindawi.com
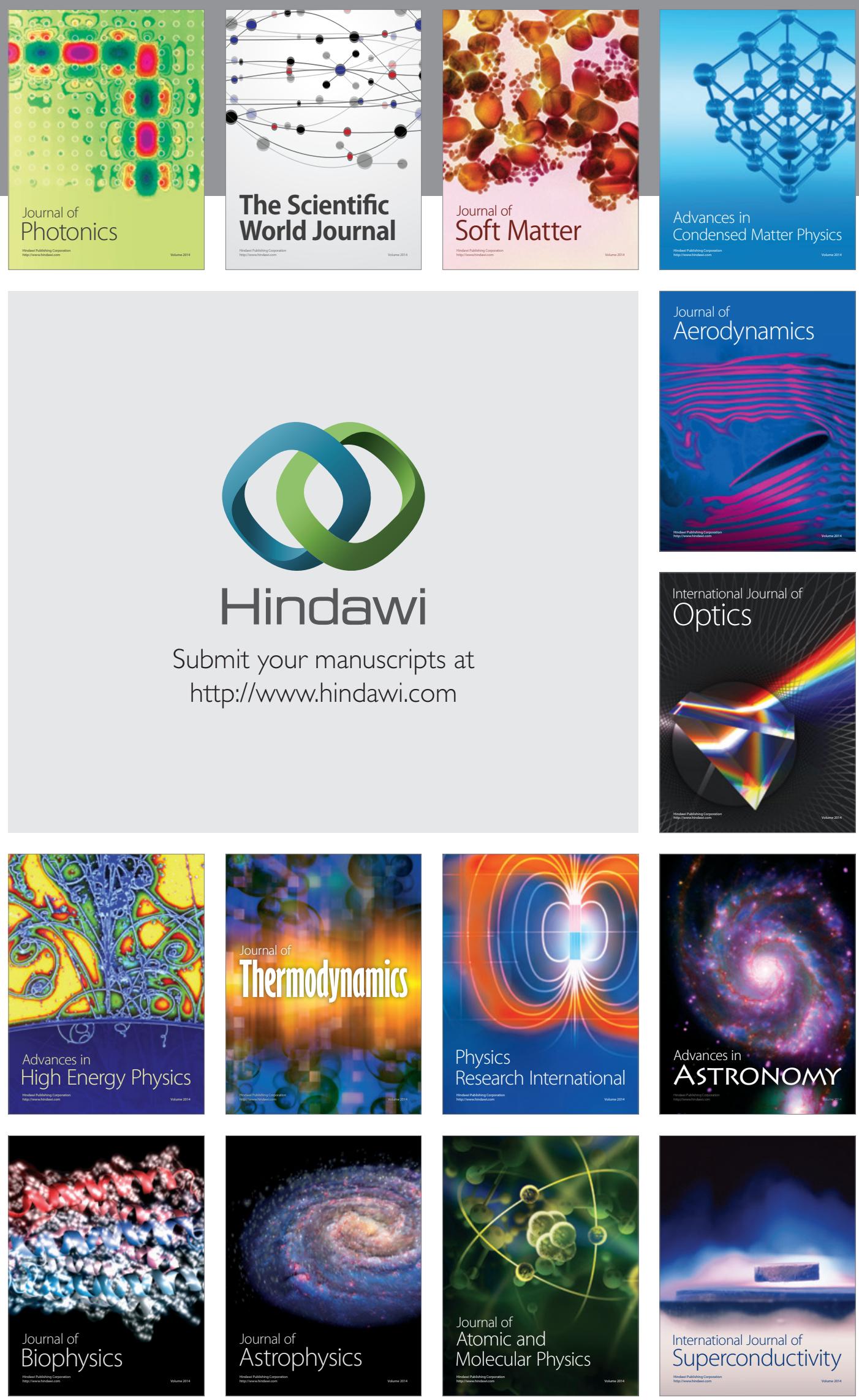
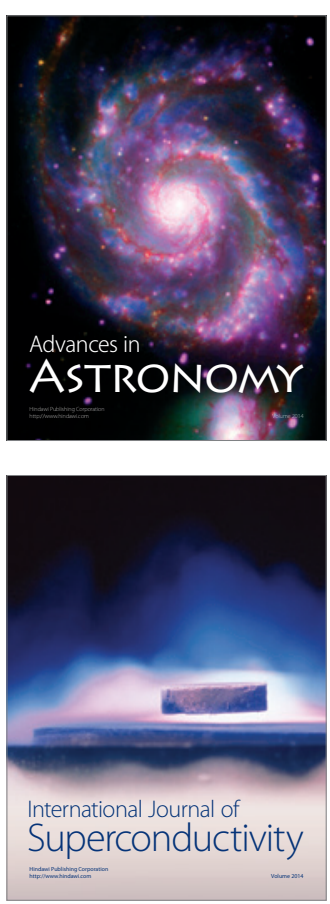